\title{
Neurofibromatosis and ectodermal dysplasia-different perspective.
}

\author{
Anubhav Chauhan ${ }^{1 *}$, Shveta Chauhan ${ }^{2}$ \\ ${ }^{1}$ Department of Ophthalmology, Dr Yashwant Singh Parmar Govt. Medical College, Nahan, District Sirmour, \\ Himachal Pradesh, India \\ ${ }^{2}$ Pine Castle, Near Mist Chamber, Khalini, Shimla 171002, Himachal Pradesh, India
}

Accepted on March 31, 2017

\section{Discussion}

Neurofibromatosis and Ectodermal Dysplasia are two systemic diseases which involve major organs of the human body and require a thorough systemic examination. During the complete evaluation of patients suffering from these diseases, often the ocular and oral examination is missed. Hence, adequate knowledge regarding these diseases need to be shared among various specialties.

A few major ocular involvements in Neurofibromatosis are keratopathy, cataract, intrascleral schwannoma, glaucoma, epiretinal membrane, retinal detachment, retinal macroaneurysm, optic nerve tumors, optic atrophy, refractive errors, strabismus, nystagmus [1], Lisch nodules (Iris hamartomas-these are virtually diagnostic of the disease and over the last few years, they have acquired a definitive role to establish or exclude the diagnosis of neurofibromatosis-type1), ptosis, proptosis, absence of orbital roof, prominent corneal nerves, choroidal hamartomas and glaucoma [2]. Oral and bony involvement in the form of periodontitis, enlarged mandibular foramen, increased coronoid notch and increased bone density is also seen [3].

The reported ophthalmologic manifestations in Ectodermal Dysplasia are strabismus, telecan thus, fused lids at birth, blepharophimosis, entropion, absence of eyelashes, bilateral eyelid cysts, agenesis of lacrimal puncta, dacryocystitis, blepharitis, conjunctivitis, deficient meibomian gland function, dry eye, corneal limbal deficiency, corneal opacity and glaucoma [4] while oral involvement is in the form of anodontia or hypodontia of the primary and/or permanent teeth, hypoplastic conical teeth, and underdevelopment of the alveolar ridges [5].

\section{References}

1. Jingyu L, Ke Y. Ocular Findings of Neurofibromatosis 2: A Case Study. J Clin Exp Ophthalmol. 2013; 43: 284.

2. Gaonker $\mathrm{CH}$, Mukherjee $\mathrm{AK}$, Pokle $\mathrm{M}$. Involvement of the eye and orbit in neurofibromatosis type 1. Indian $\mathrm{J}$ Ophthalmol. 1992;40:2-4

3. Kumar P, Dhobley A, Kumar H, et al. Type 1 neurofibromatosis with periodontal manifestations. Indian J Dent Sci. 2016;8:172-75.

4. Keklikci U, Yavuz I, Tunik S, et al. Ophthalmic Manifestations in Patients with Ectodermal Dysplasia Syndromes. Adv Clin Exp Med. 2014;23:605-10.

5. Neha Jain, Dinesh Naitam, Arti Wadkar, et al. "Prosthodontic Rehabilitation of Hereditary Ectodermal Dysplasia in an 11-Year-Old Patient with Flexible Denture: A Case Report". Case Reports in Dentistry. 2012;2012:5.

\section{*Correspondence to:}

Dr Anubhav Chauhan

Department of Ophthalmology

Nahan

Himachal Pradesh

India

Tel:+919816991482

E-mail: chauhan.anubhav2@gmail.com 